\title{
Diversity within American cassava germ plasm based on RAPD markers
}

\author{
Carlos Colombo ${ }^{1}$, Gérard Second ${ }^{2}$ and André Charrier ${ }^{3}$
}

\begin{abstract}
This work focuses on the genetic diversity of American cassava through RAPD molecular markers. The 126 genotypes studied were distributed on four geographical levels ranging from local to continental. Samples included ethnocultivars from the Santa Isabel community in the Brazilian Amazon, local cultivars collected in the State of São Paulo, native accessions from very diverse Brazilian regions, and representative accessions from the Centro Internacional de Agricultura Tropical (CIAT) core collection. Eighty-eight polymorphic bands were analyzed. Results revealed the weak genetic structure of the cassava analyzed. The pattern formed by the first two axes of the principal coordinates analysis ( $\mathrm{PCoA})$ revealed an overlapping of the São Paulo State genotype, the Brazilian group and the core collection accessions. The Santa Isabel ethnocultures formed a separate group. The weak genetic structure of cassava can be explained by the common practice of exchanging botanical material among small producers as well as by recombinations among genotypes. When the genotypes were analyzed using climatic data, the sample sites were found to be structured according to temperature and precipitation. RAPD markers proved very useful in the genetic diversity study, resulting in important implications for cassava germ plasm collections and genetic breeding.
\end{abstract}

\section{INTRODUCTION}

Cassava (Manihot esculenta Crantz, Euphorbiaceae) is one of the most important food crops in the tropics. It is a valuable source of carbohydrates, a staple in several developing nations in Africa, South America and Asia, and has the highest production potential of calories per hectare per day among tropical crops (De Bruijn and Fresco, 1989). Cassava is a shrubby species originated in the American continent approximately 3000 to 7000 years ago in several different regions: semi-arid and "cerrado" regions in Brazil (Rogers, 1972), savannas in the Orinoco River Basin between Colombia and Venezuela (Sauer, 1952), which originally belonged to adept cassava farming Aruak Indian tribes (Schmidt, 1951); hot, dry regions in Mexico and Guatemala (Rogers, 1972), and deserts along the Peruvian coast, which descended from the savannas created after glaciation (Ugent et al., 1986).

The large number of cultivars represented in the main germ plasm collections of several research centers indicates the high genetic diversity of this species. According to Martins (1994), cassava's great diversity can be explained by life history components and species dynamics as well as the horticultural practices of different Indian and traditional farmer ("caboclo" and "caiçara") communities. Cultivars are typically randomly arranged in traditional plots, which favors intercrossing. Moreover, the seeds produced in these plots disperse, fall to the ground and lie dormant for a long time.

Another possible source of new variation is mutations or interspecific crosses between wild and/or weedy Manihot species with those being cultivated. The wild and/ or weedy species may be found surrounding or inside the plot. Mutations or crosses could be fixed and propagated vegetatively (Martins, 1994).

According to Cohen et al. (1991), ex situ preserved cassavas represent about 16,000 accessions. Hershey (1994) estimated about 7,000 different cultivars. Furthermore, due to the large number of cultivars represented in collections, Boster (1985) proposed that some new cultivars were produced by human populations who traditionally consume this tubercle. The author cited new cultivars created by native populations of the Amazon Basin using visual selection criteria. Jeffrey (1968) stated that high polymorphism rates could be explained partly by vast, varied cultivation areas and conscious and unconscious breeding decisions, which accelerated this plant's evolution.

Agronomical and botanical descriptors (Pereira et al., 1992; Cury, 1993) and isoenzyme analysis (Ramirez et al., 1987; Léfèvre et al., 1993) have been developed to evaluate genetic diversity. However, a limited number of isoenzymatic systems (Pasteur et al., 1987) and botanical descriptors (Cury, 1993) are available; therefore, polymorphisms are restricted.

Molecular markers are a more stable and informative alternative to isoenzymes. According to Cohen et al. (1991), these markers can more efficiently be used to examine the genetic diversity of collections. In fact, many molecular markers are currently being used to study the genetic diversity of several different species. Marmey et al. (1994), Bonierbale et al. (1995) and Colombo (1998) have used molecular markers to study genetic diversity in cassava; however, only a limited number of genotypes were studied. 
A survey of a large number of cultivars from different regions is needed to better understand the organization and extent of genetic diversity, structuring factors, degrees of relationship between genotypes and their characterization in cassava. Consequently, conservation strategies could be developed to better exploit this germ plasm in the future. This study used RAPD molecular markers to determine the genetic diversity of American cassavas from different ecological and geographical zones.

\section{MATERIAL AND METHODS}

\section{Material}

One hundred and twenty-six assessions of $M$. esculenta were studied (Table I). Genotypes were grouped into the following four geographic levels, ranging from local to continental: a) 22 cassava ethnocultivars from a small community of "caboclos" in Santa Isabel, Amazon region (middle Negro River); b) 19 cultivars from the State of São Paulo; c) 52 accessions from different Brazilian geographical zones; d) 33 accessions from the CIAT collection selected to represent world diversity (based on a preliminary RAPD study).

\section{Methodology}

\section{DNA isolation and amplification}

DNA was isolated from silica gel dried leaf material according to the following methodology: $0.5 \mathrm{~g}$ dry leaves were ground in liquid nitrogen and transferred to $20-\mathrm{ml}$ plastic tubes. Ten milliliters of extraction buffer $(0.1 \mathrm{M}$ Tris $\mathrm{HCl}, \mathrm{pH} 8.0,1.25 \mathrm{M} \mathrm{NaCl}, 0.02 \mathrm{MEDTA}, 2 \%$ MATAB (mixed alkyltrimethylammoniun bromide)) and 1\% Bmercapto-ethanol was added just before use. After 90-min incubation at $65^{\circ} \mathrm{C}$ with slow stirring, an equal volume of chloroform/isoamylalcohol (24:1) was added twice and the resulting supernatant transferred to a clean plastic tube. RNAse ( $100 \mu \mathrm{l}$ of a $10 \mathrm{mg} / \mathrm{ml}$ solution) was added immediately after these extractions and the solution subsequently incubated at $37^{\circ} \mathrm{C}$ for $30 \mathrm{~min}$. DNA pellets were obtained by adding 0.8 vol. of isopropanol. After washing with $70 \%$ ethanol, the DNA pellet was vacuum dried and dissolved in $200 \mu \mathrm{l}$ of TE buffer $(10 \mathrm{mM}$ Tris- $\mathrm{HCl}, \mathrm{pH}$ 8.0 , containing $1 \mathrm{mM}$ EDTA). The quality and concentration of the DNA fragments were evaluated by electrophoresis in $0.8 \%$ agarose gels.

\section{PCR conditions}

PCR was carried out in a $25-\mu \mathrm{l}$ reaction containing 10 $\mathrm{mM}$ Tris- $\mathrm{HCl}, \mathrm{pH} 8.3,50 \mathrm{mM} \mathrm{KCl}, 1.5 \mathrm{mM} \mathrm{MgCl}_{2}, 0.001$ gelatin, $10 \mathrm{ng}$ template DNA, $0.4 \mu \mathrm{M}$ primer, $100 \mu \mathrm{M}$ of each dNTP and 0.5 units Taq polymerase (Appligene). DNA was amplified in a thermocycler (PTC-100 MJ Research) programmed as follows: $95^{\circ} \mathrm{C}$ for $4 \mathrm{~min}$, followed by 45 cycles of $1 \mathrm{~min}$ at $95^{\circ} \mathrm{C}, 1 \mathrm{~min}$ at $35^{\circ} \mathrm{C}$, and $2 \min$ at $72^{\circ} \mathrm{C}$, a final stage of $7 \mathrm{~min}$ at $72^{\circ} \mathrm{C}$, and then maintained at $4^{\circ} \mathrm{C}$ prior to analysis. After adding $3 \mu \mathrm{l}$ buffer $(0.5 \%$ bromophenol/blue/glycerol, 1:2:1), the amplification products were submitted to electrophoresis on $1.8 \%$ agarose gel in $1 \mathrm{X}$ TBE buffer, stained with ethidium bromide and photographed under UV light with Polaroid film.

\section{Data analysis}

Only clearly amplified polymorphic fragments were analyzed. Scores of 1 (present) or 0 (absent) were used to form a matrix. Simple matching coefficients (Sokal and Michener, 1958) were obtained to perform cluster (UPGMA) and principal coordinate analyses (PCoA). Canonical correlations (Hotelling, 1935) were used to investigate the relationship between two variable sets. The first set was represented by PCoA scores (90\% total variation) based on RAPD markers, and the second set used the average temperature and humidity values from the geographical zones of the cassava's origin. NTSYS-pc software (Rohlf, 1993) was used for the calculations. Nei's (1973) index of genetic diversity $(\mathrm{Ho})$ was calculated for the four cassava cultivar groups based on allele frequencies of dominant RAPD "loci": $H o=\sum 1 / N p$ (present) $x q$ (absent).

\section{RESULTS}

Twenty-one of the 100 primers tested were selected for their quality, quantity and reproducibility capacity of fragments amplified. Eighty-eight of the 193 bands observed were polymorphic (Table II). The average number of fragments amplified per primer and their polymorphism rate were 9.5 (ranging from 6 to 13) and 3.8 (ranging from 2 to 8 ), respectively. Size of amplified fragments varied from 300 to $2000 \mathrm{pb}$ with an average of $900 \mathrm{pb}$. An example of PCR amplification is shown in Figure 1. Only the polymorphic bright bands that were clearly amplified were analyzed and the faint bands were not scored.

Genetic similarity coefficients were calculated between all samples taken two by two. Values varied between 0.99 and 0.45 , with an average of 0.67 . No cultivars had similar profiles. Similar minimal genetic similarity values have been obtained using RAPD markers with other allogamous plants, such as 0.49 for cocoa clones (Wilde et al., 1992), 0.57 for tea (Wachira et al., 1995) and 0.45 for Picea sitchensis (Van de Ven and McNicol, 1995). Furthermore, the minimal similarity value observed among cassava cultivars of this study was greater than that of Marmey et al. (1994), which was 0.64 between the two most divergent African cassava clones.

Similarity coefficients of the four cultivar groups (Santa Isabel, São Paulo, Brazil and the World) were determined (Table III). The Santa Isabel group was significantly different from the other groups. The World group 
Table I - Plant samples used to investigate the genetic relatedness among cassava cultivars.

\begin{tabular}{|c|c|c|c|c|c|c|}
\hline No. & Code & $\begin{array}{l}\text { Name of } \\
\text { cultivar/variety }\end{array}$ & Origin/locality & Group* & Precip.* & Temp.* \\
\hline 1 & E8189-1 & Manipeba & Santa Isabel/AM/Brasil & A & 2000 & 27 \\
\hline 2 & E8190-1 & Mucura branca & Santa Isabel/AM/Brasil & A & 2000 & 27 \\
\hline 3 & E8191-2 & Maraquiri & Santa Isabel/AM/Brasil & A & 2000 & 27 \\
\hline 4 & E8192-1 & Japura & Santa Isabel/AM/Brasil & A & 2000 & 27 \\
\hline 5 & E8194-1 & Lito & Santa Isabel/AM/Brasil & A & 2000 & 27 \\
\hline 6 & E8196-1 & Fino & Santa Isabel/AM/Brasil & A & 2000 & 27 \\
\hline 7 & E8200-1 & Uapixuma & Santa Isabel/AM/Brasil & A & 2000 & 27 \\
\hline 8 & E8201-1 & Mucura Roxa & Santa Isabel/AM/Brasil & A & 2000 & 27 \\
\hline 9 & E8203-1 & Taboquinha & Santa Isabel/AM/Brasil & A & 2000 & 27 \\
\hline 10 & E8204-1 & Samaúma & Santa Isabel/AM/Brasil & A & 2000 & 27 \\
\hline 11 & E8205-1 & Barcelos & Santa Isabel/AM/Brasil & A & 2000 & 27 \\
\hline 12 & E8207-2 & Baixinha & Santa Isabel/AM/Brasil & A & 2000 & 27 \\
\hline 13 & E8208-1 & Juruna & Santa Isabel/AM/Brasil & A & 2000 & 27 \\
\hline 14 & E8209-1 & unnamed & Santa Isabel/AM/Brasil & A & 2000 & 27 \\
\hline 15 & E8210-1 & unnamed & Santa Isabel/AM/Brasil & A & 2000 & 27 \\
\hline 16 & E8211-1 & unnamed & Santa Isabel/AM/Brasil & A & 2000 & 27 \\
\hline 17 & E8215-1 & Seis Meses Branca & Santa Isabel/AM/Brasil & A & 2000 & 27 \\
\hline 18 & E8214-1 & Malave & Santa Isabel/AM/Brasil & A & 2000 & 27 \\
\hline 19 & E8216-2 & Esteio Branco & Santa Isabel/AM/Brasil & A & 2000 & 27 \\
\hline 20 & E8221 & Buia 3 & Santa Isabel/AM/Brasil & A & 2000 & 27 \\
\hline 21 & E8223 & Buia 5 & Santa Isabel/AM/Brasil & A & 2000 & 27 \\
\hline 22 & E8225 & Buia 7 & Santa Isabel/AM/Brasil & A & 2000 & 27 \\
\hline 23 & F1089 & Lavoura & Monteiro Lobato/SP/Brasil & B & 1250 & 24 \\
\hline 24 & F1117 & Santista Branca & Ubatuba/SP/Brasil & B & 2000 & 22 \\
\hline 25 & F1135 & Pão de Ló & Maresias/SP/Brasil & B & 1750 & 22 \\
\hline 26 & F1153 & Cacau I & Praia Grande/SP/Brasil & B & 1850 & 22 \\
\hline 27 & F1162 & unnamed & Peruibi/SP/Brasil & B & 1850 & 22 \\
\hline 28 & F2023 & unnamed & Jacupiranga/SP/Brasil & B & 1650 & 22 \\
\hline 29 & F2030 & Pão do Céu II & Iporanga/SP/Brasil & B & 1650 & 22 \\
\hline 30 & F2072 & unnamed & Itapetininga/SP/Brasil & B & 1350 & 20 \\
\hline 31 & F3039 & Canela de Urubu & Franca/SP/Brasil & B & 1500 & 20 \\
\hline 32 & F3075 & unnamed & Barretos/SP/Brasil & B & 1350 & 21 \\
\hline 33 & F4025 & Santa Catarina & Catanduva/SP/Brasil & B & 1250 & 22 \\
\hline 34 & F4048 & unnamed & Fernandópolis/SP/Brasil & B & 1250 & 22 \\
\hline 35 & F4072 & unnamed & Araçatuba/SP/Brasil & B & 1250 & 22 \\
\hline 36 & F4113 & Mato Grosso & Bariri/SP/Brasil & B & 1250 & 20 \\
\hline 37 & F4130 & unnamed & Sta Bárbara D’Oeste/SP/Brasil & B & 1250 & 20 \\
\hline 38 & F5075 & Vassourinha XII & Ouro Verde/SP/Brasil & B & 1250 & 22 \\
\hline 39 & F5096 & Amarela VI & Pirapozinho/SP/Brasil & B & 1250 & 22 \\
\hline 40 & F5115 & unnamed & Assis/SP/Brasil & B & 1350 & 21 \\
\hline 41 & F5129 & Vassourinha XIV & Chavantes/SP/Brasil & B & 1350 & 21 \\
\hline 42 & SRT1 & Vassourinha Pta & Unknown/Brasil & $\mathrm{C}$ & - & - \\
\hline 43 & SRT59 & Branca de Santa Catarina & Volta Grande/SC/Brasil & $\mathrm{C}$ & 2150 & 17 \\
\hline 44 & SRT120 & Santa & Ubatuba/SP/Brasil & $\mathrm{C}$ & 2000 & 22 \\
\hline 45 & SRT454 & Guaxupé & Guaxupé/MG/Brasil & $\mathrm{C}$ & 1500 & 19 \\
\hline 46 & SRT521 & Carape II & Unknown/Brasil & $\mathrm{C}$ & 1500 & 18 \\
\hline 47 & SRT1012 & Guaxo & Araquari/SC/Brasil & $\mathrm{C}$ & 1750 & 20 \\
\hline 48 & SRT1105 & Mico & Rio do Sul/SC/Brasil & $\mathrm{C}$ & 1500 & 19 \\
\hline 49 & SRT1116 & Cigana Preta & Cruz das Almas/BA/Brasil & $\mathrm{C}$ & 1100 & 24 \\
\hline 50 & SRT1196 & Cavalo & Brasília/DF/Brasil & $\mathrm{C}$ & 1600 & 20 \\
\hline 51 & SRT1197 & Paiza & Brasília/DF/Brasil & $\mathrm{C}$ & 1600 & 20 \\
\hline 52 & SRT1214 & Unha & São Mateus/ES/Brasil & $\mathrm{C}$ & 1250 & 24 \\
\hline 53 & SRT1256 & unnamed & Venceslau Brás/PR/Brasil & $\mathrm{C}$ & 1350 & 19 \\
\hline 54 & SRT1273 & Azulona & Gentil do Ouro/BA/Brasil & $\mathrm{C}$ & 750 & 22 \\
\hline 55 & SRT1275 & Aipim Branco & Gentil do Ouro/BA/Brasil & $\mathrm{C}$ & 750 & 22 \\
\hline 56 & SRT1293 & unnamed & Itumbiara/GO/Brasil & $\mathrm{C}$ & 1600 & 20 \\
\hline 57 & SRT1312 & Izabel de Souza II & São João/PE/Brasil & $\mathrm{C}$ & 750 & 21 \\
\hline 58 & SRT1313 & Cambadinha & Tabira/PE/Brasil & $\mathrm{C}$ & 750 & 22 \\
\hline 59 & SRT1315 & Cravo & Moreno/PE/Brasil & $\mathrm{C}$ & 1800 & 25 \\
\hline 60 & SRT1316 & Pacare & São João/PE/Brasil & $\mathrm{C}$ & 750 & 21 \\
\hline 61 & SRT1317 & Pipoca & Afogados do Ingazeira/PE/Brasil & $\mathrm{C}$ & 750 & 23 \\
\hline 62 & SRT1318 & Pai Antonio & São João/PE/Brasil & $\mathrm{C}$ & 750 & 21 \\
\hline 63 & SRT1319 & Lagoa & São João/PE/Brasil & $\mathrm{C}$ & 750 & 21 \\
\hline
\end{tabular}


Table I - Continued

\begin{tabular}{|c|c|c|c|c|c|c|}
\hline No. & Code & $\begin{array}{l}\text { Name of } \\
\text { cultivar/variety }\end{array}$ & Origin/locality & Group* & Precip.* & Temp.* \\
\hline 64 & SRT1326 & Mandiocaba & Belém/PA/Brasil & $\mathrm{C}$ & 2350 & 26 \\
\hline 65 & SRT1327 & Sutinga III & Belém/PA/Brasil & $\mathrm{C}$ & 2350 & 26 \\
\hline 66 & SRT1328 & Uapixuna & Belém/PA/Brasil & $\mathrm{C}$ & 2350 & 26 \\
\hline 67 & SRT1333 & Amarela & Coxim/MS/Brasil & $\mathrm{C}$ & 1250 & 24 \\
\hline 68 & SRT1336 & Olho Roxo & Jaceara/MS/Brasil & $\mathrm{C}$ & 1500 & 24 \\
\hline 69 & SRT1337 & Pão XIII & Jaceara/MS/Brasil & $\mathrm{C}$ & 1500 & 24 \\
\hline 70 & SRT1338 & Paraguainha & Jaceara/MS/Brasil & $\mathrm{C}$ & 1500 & 24 \\
\hline 71 & SRT1340 & Buião & São Francisco/MG/Brasil & $\mathrm{C}$ & 1000 & 24 \\
\hline 72 & SRT1341 & Bambu & São Francisco/MG/Brasil & $\mathrm{C}$ & 1000 & 24 \\
\hline 73 & SRT1342 & Manteiga IV & São Francisco/MG/Brasil & $\mathrm{C}$ & 1000 & 24 \\
\hline 74 & SRT1344 & Jaburu & Ceará State/Brasil & $\mathrm{C}$ & - & - \\
\hline 75 & SRT1345 & Saracura & Santa Cruz/RJ/Brasil & $\mathrm{C}$ & 1350 & 24 \\
\hline 76 & SRT1351 & Apronta a Mesa & Rio Grande do Sul State/Brasil & $\mathrm{C}$ & - & - \\
\hline 77 & BGM276 & IAC 5-36 & Crossing/Brasil & $\mathrm{C}$ & - & - \\
\hline 78 & BGM243 & EAB-105 & Crossing/Brasil & $\mathrm{C}$ & - & - \\
\hline 79 & BGM95 & Paraguainha & Canavierias/BA/Brasil & $\mathrm{C}$ & 1800 & 24 \\
\hline 80 & BGM82 & Branca SC & Unknown/Brasil & $\mathrm{C}$ & - & - \\
\hline 81 & BGM128 & Embuzeiro & Alagoa/RS/Brasil & $\mathrm{C}$ & 1600 & 20 \\
\hline 82 & BGM134 & Palmeira Preta & Irara/BA/Brasil & $\mathrm{C}$ & 1500 & 24 \\
\hline 83 & BGM252 & Manteiga & MacedoCosta/BA/Brasil & $\mathrm{C}$ & - & - \\
\hline 84 & BGM42 & Brava de Padua & unknown/Brasil & $\mathrm{C}$ & - & - \\
\hline 85 & BGM21 & Cachimbo & unknown/Brasil & $\mathrm{C}$ & 2350 & 26 \\
\hline 86 & BGM739 & Cambraia II & unknown/Brasil & $\mathrm{C}$ & 1250 & 24 \\
\hline 87 & BGM32 & Cidade Rica & Tangara/RN/Brasil & $\mathrm{C}$ & 1200 & 24 \\
\hline 88 & BGM1269 & Engana Ladrão & Petrolina/PE/Brasil & $\mathrm{C}$ & 600 & 26 \\
\hline 89 & BGM441 & Taquari-SRT 1099 & Rio Grande do Sul State/Brasil & $\mathrm{C}$ & 1500 & 18 \\
\hline 90 & BGM1014 & Mico & Itajaí/RS/Brasil & $\mathrm{C}$ & 1600 & 19 \\
\hline 91 & BGM1013 & Mandim Branca & Itajaí/RS/Brasil & $\mathrm{C}$ & 1600 & 19 \\
\hline 92 & BGM81 & Mameluca & Pará State/Brasil & $\mathrm{C}$ & 2350 & 26 \\
\hline 93 & BGM19 & Xingu & Belém/PA/Brasil & $\mathrm{C}$ & 2350 & 26 \\
\hline 94 & ARG11 & Duro do Valle 30 & Argentina & $\mathrm{D}$ & 1000 & 21 \\
\hline 95 & BOL3 & Rasada de Bolivia & Bolivia & $\mathrm{D}$ & 1400 & 25 \\
\hline 96 & BRA12 & unknown & Brasil & $\mathrm{D}$ & - & - \\
\hline 97 & BRA110 & Pangola & Araua/Sergipel & $\mathrm{D}$ & - & - \\
\hline 98 & BRA383 & Vassourão & Brasil & $\mathrm{D}$ & - & - \\
\hline 99 & BRA881 & Branca de Santa Catarina & Cruz das Almas/BA & $\mathrm{D}$ & 1100 & 24 \\
\hline 100 & COL22 & Uvita & Cordoba-Ayapel & $\mathrm{D}$ & 1500 & 25 \\
\hline 101 & COL1438 & Llanera & Colombia & $\mathrm{D}$ & 1800 & 27 \\
\hline 102 & COL1468 & Mantiqueira & Colombia & $\mathrm{D}$ & - & - \\
\hline 103 & COL1505 & unknown & Venezuela & $\mathrm{D}$ & - & - \\
\hline 104 & COL1522 & Algodonera Amarilla & Cauca-Morales/Colombia & $\mathrm{D}$ & 1500 & 23 \\
\hline 105 & COL1684 & unknown & Colombia & $\mathrm{D}$ & - & - \\
\hline 106 & COL2061 & Regional morada & Popayan/Colombia & $\mathrm{D}$ & 1910 & 17 \\
\hline 107 & COL2066 & Chiroza Gallinaza & Caicedonia/Colombia & $\mathrm{D}$ & - & - \\
\hline 108 & COL2215 & Venezolana 1 & Pivijay/Colombia & $\mathrm{D}$ & 1000 & 28 \\
\hline 109 & CR32 & Yuca Mangi & Costa Rica & $\mathrm{D}$ & 2500 & - \\
\hline 110 & CUB51 & Pinera & Cuba & $\mathrm{D}$ & 1400 & 26 \\
\hline 111 & CUB74 & Señorita & Cuba & $\mathrm{D}$ & 1400 & 26 \\
\hline 112 & ECU41 & De tres meses & Manabi-Chone/Equador & D & 370 & 30 \\
\hline 113 & ECU82 & Blanca & Loja-Loja/Equador & $\mathrm{D}$ & 1500 & 20 \\
\hline 114 & HMC1 & unknown & Unkown & D & - & - \\
\hline 115 & IND33 & unknown & Bogor/Malaysia & $\mathrm{D}$ & 2600 & 27 \\
\hline 116 & MAL2 & Black Twig & Malaysia & $\mathrm{D}$ & - & - \\
\hline 117 & MAL48 & Red Twig & Kuala Lumpur/Malaysia & $\mathrm{D}$ & 2400 & 26 \\
\hline 118 & MEX59 & unknown & Chiapas/Mexique & $\mathrm{D}$ & - & - \\
\hline 119 & NGA2 & Hybrid (TMS30572) & Ibadam/Nigeria & $\mathrm{D}$ & - & - \\
\hline 120 & PAN51 & Unknown & Ocu-Ocu/Panama & $\mathrm{D}$ & 3000 & 27 \\
\hline 121 & PAR110 & Tacuara Sayyu & Colonia Liberation/Paraguai & $\mathrm{D}$ & 1400 & 23 \\
\hline 122 & PTR19 & NO.9588 & Puerto Rico & $\mathrm{D}$ & 1550 & 26 \\
\hline 123 & TAI1 & Rayong 1 & Bangkhen-Bangkok/Thailand & $\mathrm{D}$ & 1500 & 28 \\
\hline 124 & THAI2 & unknown & Thailand & $\mathrm{D}$ & - & - \\
\hline 125 & VEN25 & Querepa Amarga & San Rafael Maraimo/Venezuela & $\mathrm{D}$ & 2500 & 22 \\
\hline 126 & VEN45-A & unknown & Venezuela & $\mathrm{D}$ & - & - \\
\hline
\end{tabular}

* Cassava germplasm from Santa Isabel (A), São Paulo (B), Brazil (C) and World collection (D) and local average precipitation (Precip.) and temperature (Temp.). 
Table II - Number and frequency of RAPD polymorphism products amplified by the primer in cassava.

\begin{tabular}{|c|c|c|c|c|c|c|c|}
\hline \multirow[t]{2}{*}{$\begin{array}{l}\text { Primer } \\
\text { (Operon) }\end{array}$} & \multirow{2}{*}{$\begin{array}{l}\text { Number of } \\
\text { RAPD products } \\
\text { by primer }\end{array}$} & \multirow{2}{*}{$\begin{array}{c}\text { Number of } \\
\text { polymorphic RAPD } \\
\text { products by primer }\end{array}$} & \multirow[t]{2}{*}{$\begin{array}{l}\text { Polymorphic product } \\
\text { percentage }\end{array}$} & \multicolumn{4}{|c|}{$\begin{array}{l}\text { Number of polymorphic RAPD } \\
\text { products by groups }\end{array}$} \\
\hline & & & & Santa Isabel & São Paulo & Brazil & World \\
\hline H6 & 10 & 7 & 70 & 7 & 7 & 7 & 7 \\
\hline H7 & 7 & 2 & 29 & 1 & 2 & 2 & 2 \\
\hline I1 & 9 & 3 & 33 & 3 & 3 & 3 & 3 \\
\hline I8 & 7 & 2 & 29 & 2 & 2 & 2 & 2 \\
\hline J9 & 11 & 2 & 18 & 1 & 2 & 2 & 1 \\
\hline J10 & 11 & 4 & 36 & 4 & 2 & 2 & 3 \\
\hline K12 & 11 & 5 & 45 & 4 & 4 & 4 & 5 \\
\hline K14 & 10 & 7 & 70 & 5 & 5 & 6 & 7 \\
\hline L7 & 13 & 6 & 46 & 6 & 6 & 6 & 6 \\
\hline M10 & 10 & 5 & 50 & 3 & 3 & 3 & 5 \\
\hline M12 & 6 & 3 & 50 & 2 & 2 & 2 & 3 \\
\hline N5 & 9 & 5 & 56 & 3 & 5 & 5 & 5 \\
\hline N20 & 9 & 5 & 56 & 4 & 3 & 3 & 5 \\
\hline X5 & 7 & 5 & 71 & 5 & 2 & 3 & 4 \\
\hline Y11 & 9 & 4 & 44 & 3 & 3 & 4 & 4 \\
\hline Y14 & 11 & 3 & 27 & 3 & 3 & 3 & 3 \\
\hline Y16 & 6 & 4 & 67 & 3 & 3 & 3 & 4 \\
\hline $\mathbf{Z 4}$ & 11 & 8 & 73 & 7 & 6 & 7 & 8 \\
\hline $\mathrm{Z6}$ & 7 & 3 & 43 & 2 & 3 & 3 & 3 \\
\hline Z9 & 9 & 2 & 22 & 1 & 2 & 2 & 2 \\
\hline $\mathbf{Z 1 7}$ & 10 & 3 & 30 & 2 & 3 & 3 & 3 \\
\hline Totals & 193 & 88 & 46 & 71 & 71 & 75 & 85 \\
\hline \multicolumn{4}{|c|}{ \% of polymorphism } & 81 & 81 & 85 & 97 \\
\hline
\end{tabular}

\section{$\begin{array}{llll}\text { S. Paulo } & \text { Brazil } & \text { World } & \text { Santa Isabel }\end{array}$}
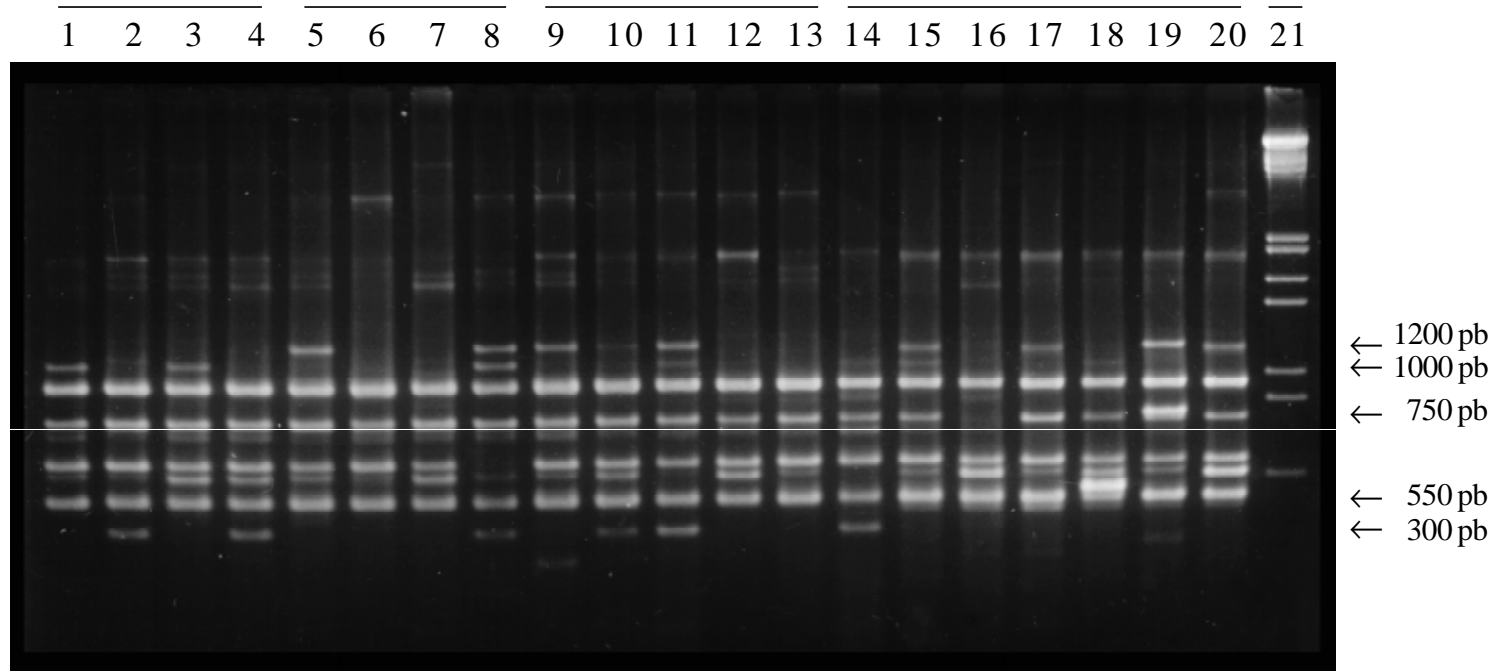

Figure 1 - Example of cassava RAPD amplification (primer N20 Operon) in agarose gel 1.8\%. Only the clearly amplified polymorphic bright bands were analyzed (arrows signal the length of these fragments in pairs of bases). The faint bands were not scored.

was the most diverse. Surprisingly, similar results were found for the cultivars from the State of São Paulo. Local cultivars collected in this state were almost as diverse as those of the world collection.

A dendrogram of genetic relationships was established from the similarity coefficient matrix (Figure 2). The hierarchical agglomeration of cultivars was constructed according to the UPGMA method. No strong structure was found among major groups. Nevertheless, most genotypes regrouped on three main branches (A, B and C), or a fourth branch (D), consisting of four samples (Tai1, Mal48, Mal2 and BGM739). All local cultivars from the Amazon (E) belonged to branch A. Cultivars from the State of São Paulo (F), all of Brazil (SRT and BGM) as well as those from the CIAT core collection were well distributed on nearly all branches of the dendrogram. 
The first two axes of the principal coordinate analysis (PCoA) (Figure 3) were used to structure the four geographically different cassava groups. The percentage of total inertia of axes 1 and 2 was about 14\%. Distribution of three of the four cassava groups (São Paulo, Brazil and World) overlapped. Neither axis 1 nor 2 was capable of distinguishing these groups. However, the Santa Isabel group was defined by axis 1 .

Shannon genetic diversity indices $(\mathrm{Ho})$ were calculated with all RAPD markers (Table IV). A comparison of the averages revealed significantly less genetic diversity in the Santa Isabel cultivar that the other groups. World collection cultivars were the most diverse (0.93), followed by the State of São Paulo and Brazil, both with 0.91. However, when these differences were examined by comparing averages, they were not statistically significant.
Canonical analysis (Figure 4) was performed on 90 of the 126 cassava genotypes that had climatic data available (Table I). In addition, factors calculated by PCoA were based on 88 RAPD markers. The first discriminant function was statistically significant by itself $(\mathrm{R}=0.82, \mathrm{P}<$ 0.0052 ). The two variable groups studied, climatic data and PCoA factors, were statistically correlated. Root coefficients were -0.25 and 0.92 for the variables precipitation and temperature, respectively.

Discriminant analysis was performed on the precipitation data of 90 cassava cultivars (Figure 5). Each variable was correlated with PCoA factors obtained with RAPD markers. Cultivars distributed by the two discriminant functions structured around the variable precipitation. Group P1 consisted of cultivars from weak precipitation zones, group $\mathrm{P} 2$ cultivars from average precipita-

Table III - Simple matching coefficient values for cassava cultivar groups: Santa Isabel local cultivars, São Paulo State cultivars, accessions of the Brazilian group and accessions of the World collection.

\begin{tabular}{|lccccc|}
\hline & Total & Santa Isabel (1) & São Paulo (2) & Brazil (3) & World (4) \\
\hline Number of cultivars & 126 & 22 & 19 & 52 & 33 \\
Maximal & 0.987 & 0.987 & 0.896 & 0.909 & 0.948 \\
Minimal & 0.468 & 0.519 & 0.532 & 0.519 & 0.494 \\
Average & $\mathbf{0 . 6 6 3}$ & $\mathbf{0 . 7 1 7}$ & $\mathbf{0 . 6 7 7}$ & $\mathbf{0 . 6 7 9}$ & $\mathbf{0 . 6 6 3}$ \\
Standard deviation & 0.005 & 0.006 & 0.007 & 0.005 & 0.006 \\
CV & 0.089 & 0.093 & 0.102 & 0.081 & 0.096 \\
\hline
\end{tabular}

Comparison of averages by the $t$-test (Student-Fisher)

$t 1 \times 2=2.25(\mathrm{P}<0.05) \quad t 1 \times 3=4.88(\mathrm{P}<0.05) \quad t 1 \times 4=4.62(\mathrm{P}<0.05) \quad t 2 \times 3=0.02 n s \quad t 2 \times 4=1.22 n s \quad t 3 \times 4=1.13 n s$

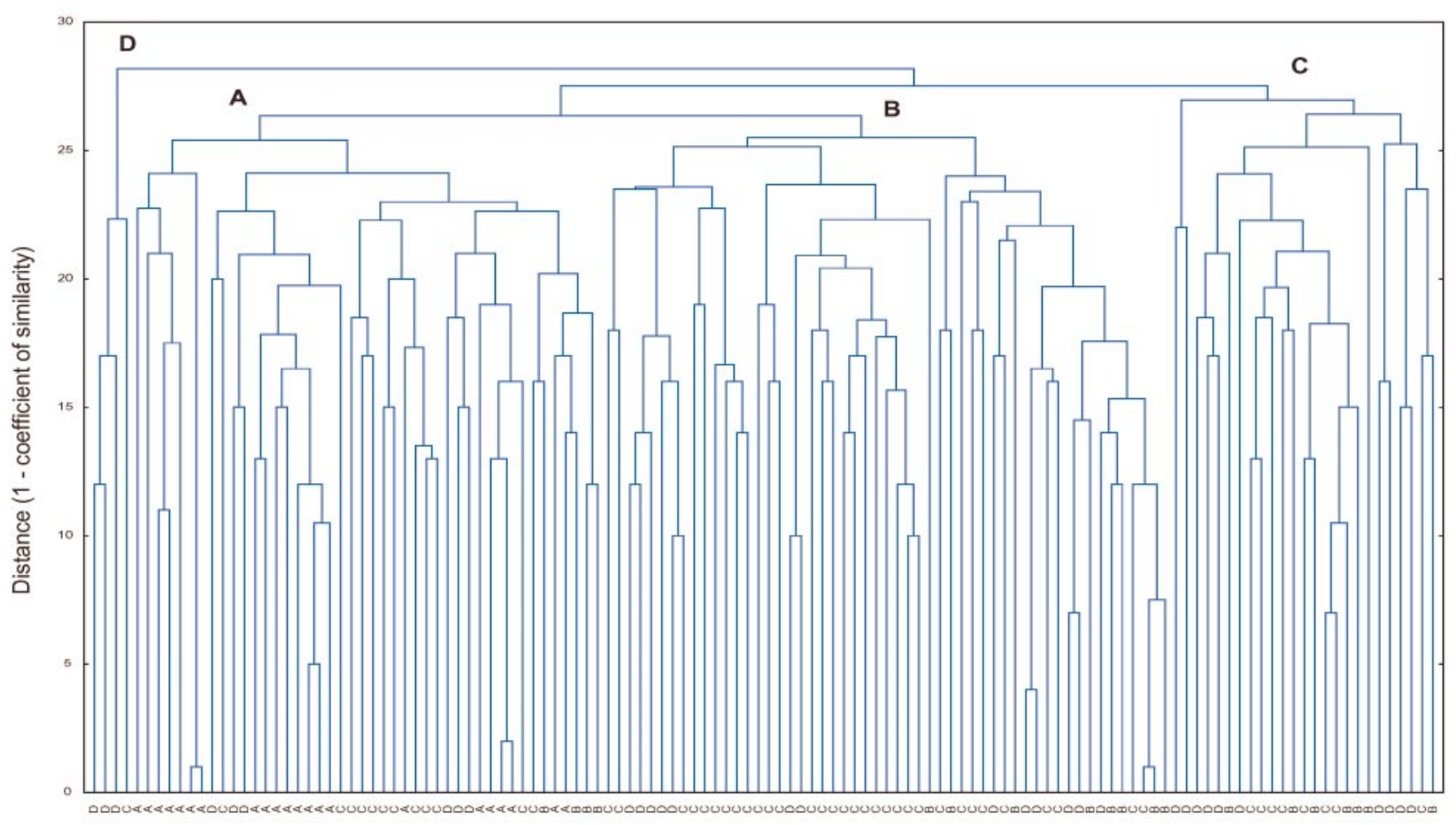

Figure 2 - Dendrogram based on UPGMA model calculated from genetic distances among American cassava genotypes from Santa Isabel (A), São Paulo (B), Brazil (C) and world collection (D), employing 88 RAPD markers. 


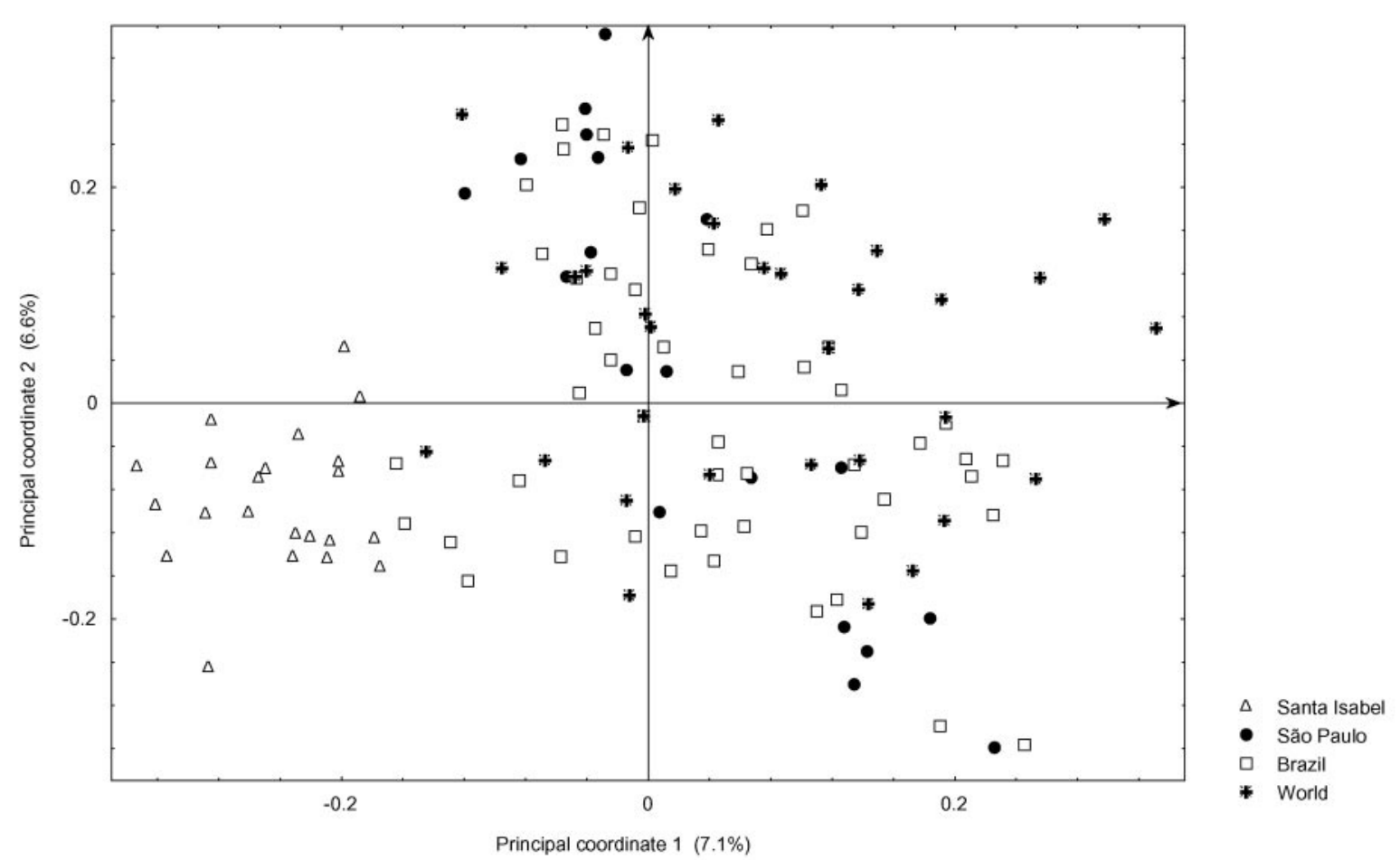

Figure 3 - Plot defined by the first two principal coordinate analysis factors based on genetic similarities (simple matching coefficients) of 126 cassava genotypes. Similarity coefficients were calculated with 88 RAPD markers.

Table IV - Shannon genetic index for the four cassava cultivar groups and comparisons of their averages by $t$-test (Student-Fisher).

\begin{tabular}{|c|c|c|}
\hline & Shannon diversity $(\mathrm{Ho})$ & Average comparison $(t)$ \\
\hline $\begin{array}{l}\mathrm{Ho}{ }_{\text {Santa Isabel (1) }} \\
\mathrm{Ho}{ }_{\text {São Paulo (2) }} \\
\mathrm{Ho}{ }_{\text {Brazil (3) }} \\
{ }_{\text {World (4) }}\end{array}$ & $\begin{array}{l}0.80 \\
0.91 \\
0.91 \\
0.93\end{array}$ & $\begin{array}{l}t_{1 \times 2}=5.78 * * \\
t_{1 \times 3}=5.36 * * \\
t_{1 \times 4}=6.53 * * \\
t_{2 \times 3}=0.00 \mathrm{~ns} \\
t_{2 \times 4}=1.04 \mathrm{~ns} \\
t_{3 \times 4}=0.97 \mathrm{~ns}\end{array}$ \\
\hline
\end{tabular}

ns, Nonsignificant. **Statistically significant at $1 \%$.

tion zones and group P3 cultivars from strong precipitation zones. Group P1 was composed of cultivars from northeastern Brazil, the most arid zone in the country, as well as cultivar ECU 41, the cultivar from the area with the least precipitation among the 90 cultivars included in this study. Group P3 was primarily formed by cultivars from northern Brazil, the Brazilian coast and Central American. Group P2 united cultivars from average precipitation zones, primarily central and southern Brazil.

Discriminant analysis was also performed on the variable temperature (Figure 6). As in the previous analysis, cultivars structured around the variable temperature. All groups, except groups $\mathrm{T} 1\left(<19.9^{\circ} \mathrm{C}\right)$ and $\mathrm{T} 2\left(20^{\circ} \mathrm{C}\right.$ $\left.22.9^{\circ} \mathrm{C}\right)$, were significantly different $(\mathrm{P}=0.05)$. All groups except the mainly equatorial group $\mathrm{T} 4$, were composed of cultivars from several different regions.

\section{DISCUSSION}

Cassava has high genetic diversity, which indicates a large genetic base. However, most of the polymorphic RAPD markers used in this study did not reveal much about cassava's genetic structure. It can be supposed that most RAPD markers were well distributed throughout the entire genome. Moreover, these markers revealed an important degree of homoplasy for the structuring of cassava, according to their degree of relationship. These results illustrate that RAPD markers could be useful for structuring the genetic diversity of collections, assessment or formation of a core collection, and especially construction of a genetic map. In addition, these markers are considered valid for identification or characterization of cultivars, establishment of genetic distance between genotypes or identification of identical lines in a collection of clones, as shown in Colombo et al. (1998).

Diversity of local cultivars from the State of São Paulo was identical to that of accessions from the whole country. This result is interesting because São Paulo represents only $1 / 32$ of Brazilian territory. Cassava cultivars collected in this state came from small producers whose produce was destined for domestic consumption. Normally these cultivars have distinct agronomic features based on the producer's preference, which can explain the great diversity found among these cultivars.

Diversity among cultivars distributed throughout Brazil (Brazil group) was also just a little less than acces- 


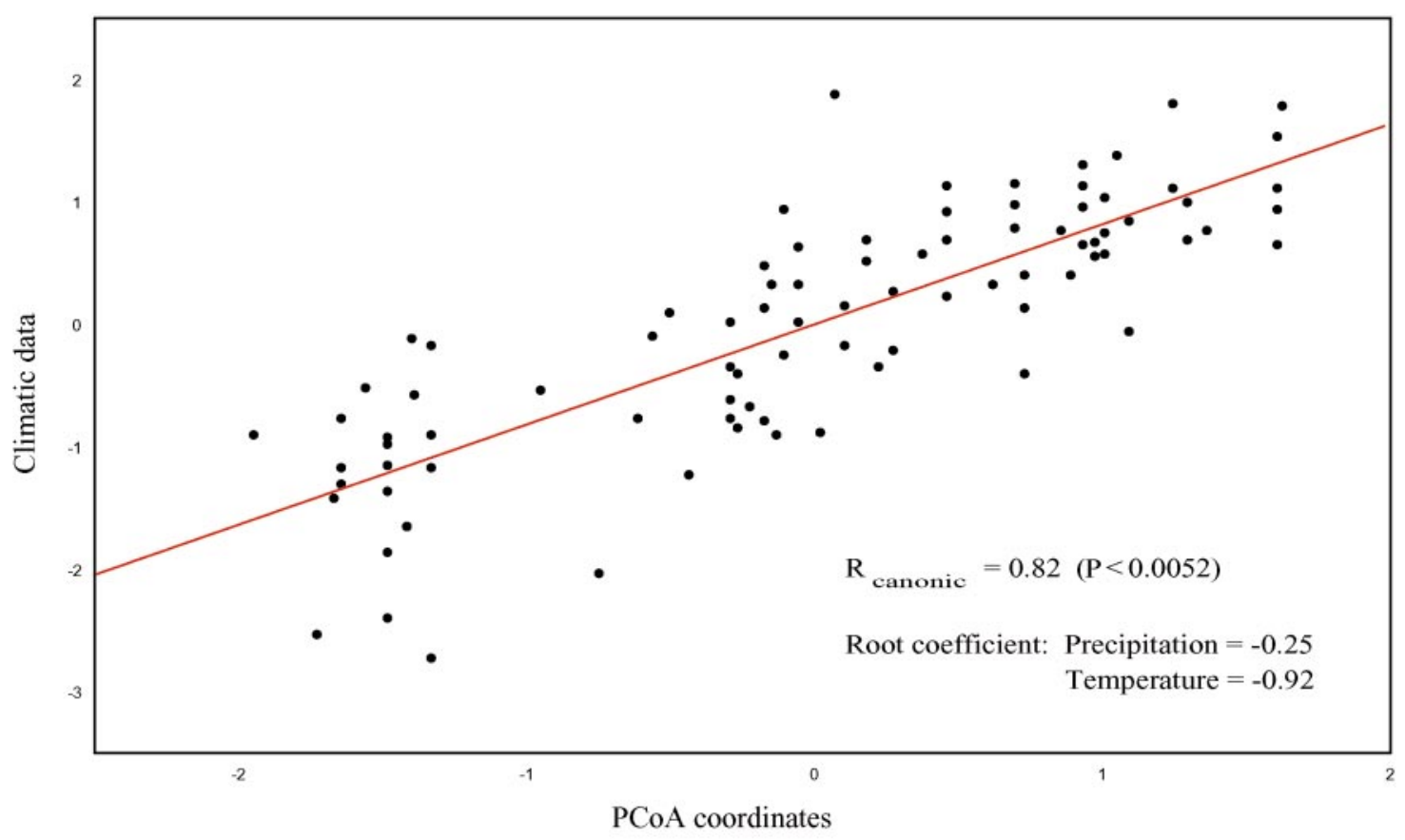

Figure 4 - Canonical analysis of climatic data (temperature and precipitation) from 90 cassava cultivar sites and principal coordinate analysis (PCoA) factors, calculated with 88 RAPD markers.

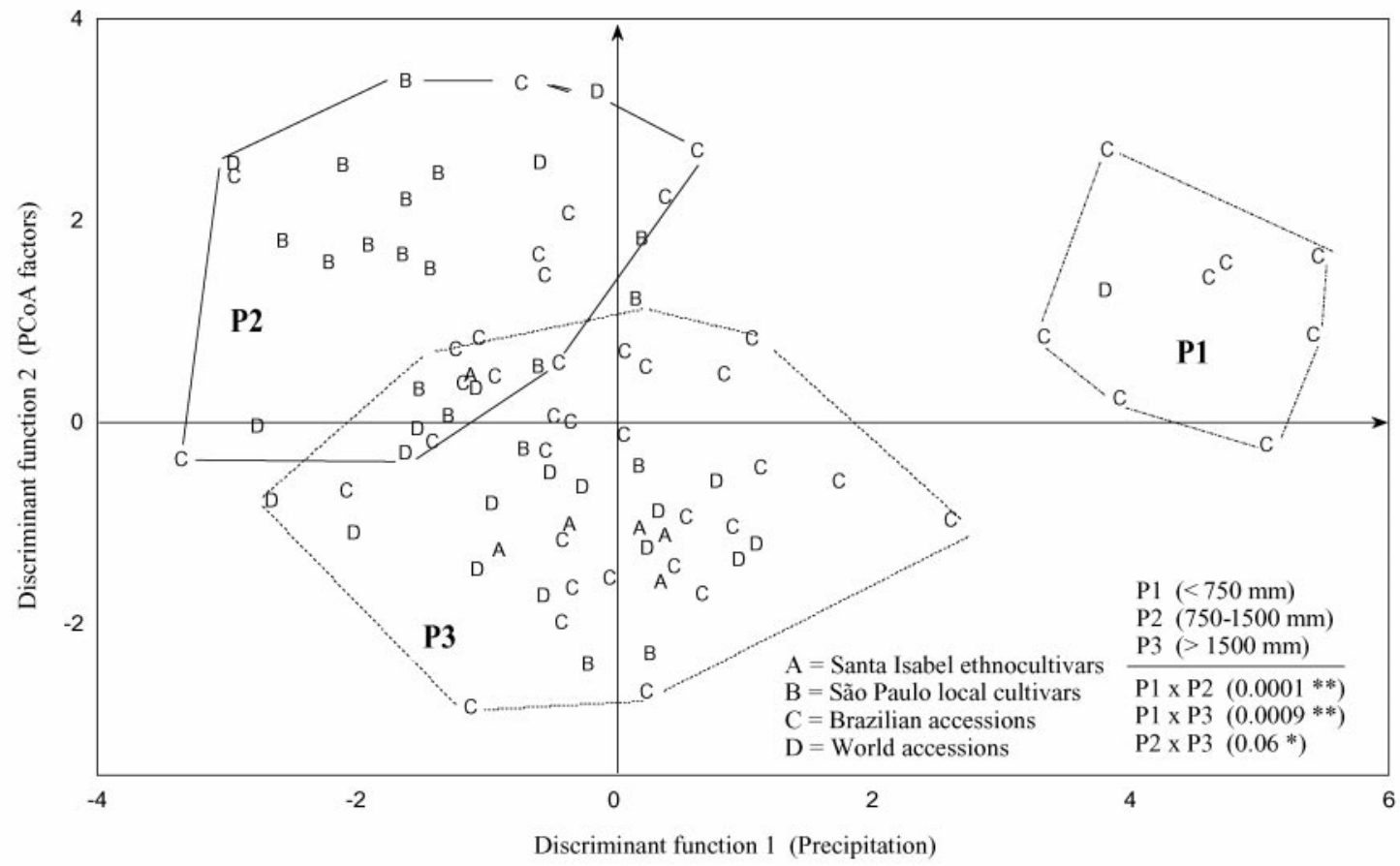

Figure 5 - Scattering of 90 cassava cultivars on a plot defined by discriminant function 1, based on precipitation (P1 to P3) of cultivar's place of origin, and discriminant function 2 , based on the first 40 principal coordinate analysis (PcoA) factors. *** Statistically significant at $5 \%$ and $1 \%$, respectively.

sions representing the world collection, which was composed of American accessions. This result supports the hypothesis that Brazil being is a center of cassava diversity, which corroborates Nassar's report (1978). It has continental dimensions with diverse ecological condi- tions and is historically the largest cassava producer and consumer worldwide.

The large degree of genetic diversity of ethnocultivars from Santa Isabel was surprising. This considerable genetic diversity could be explained by the Indian agricul- 


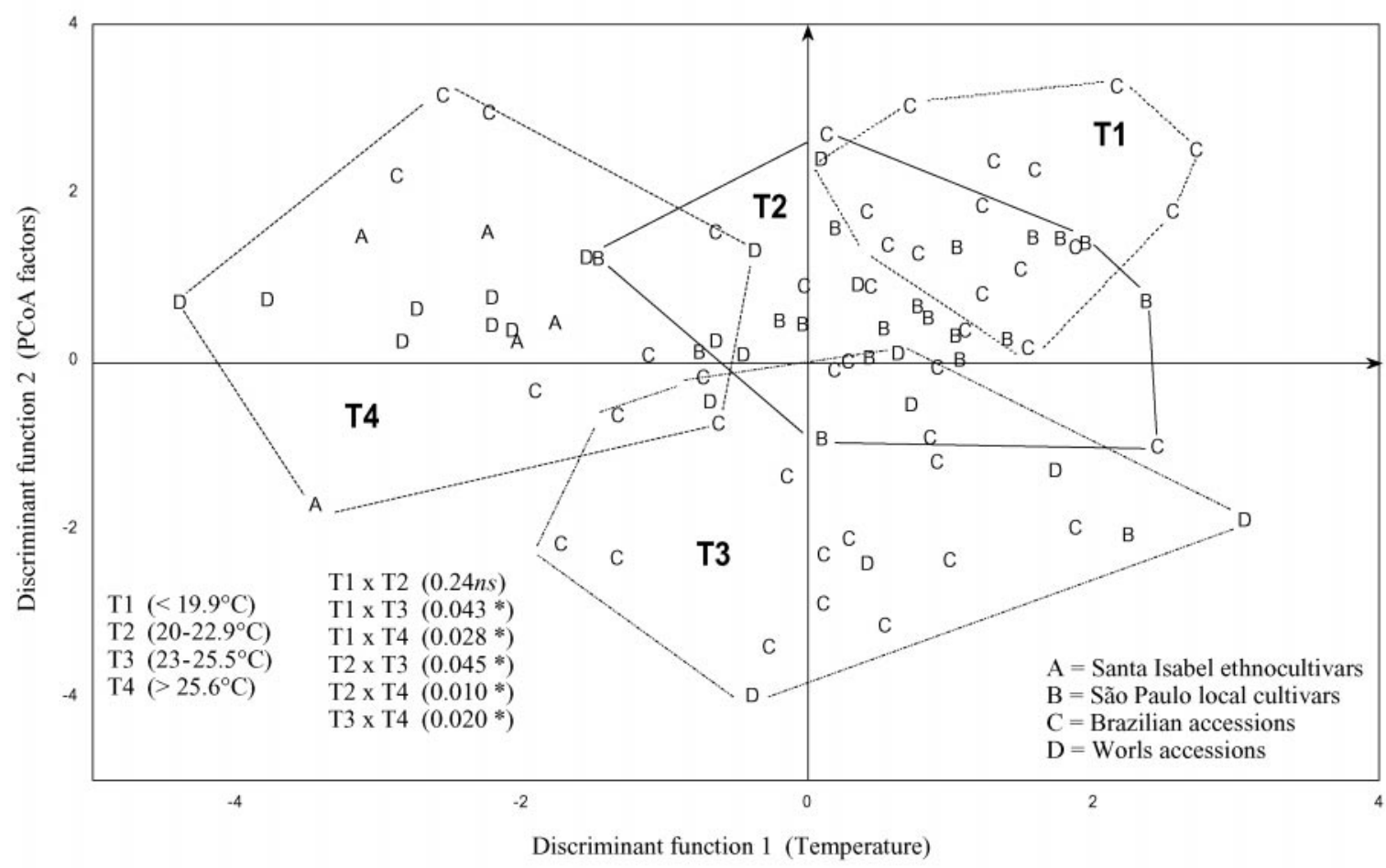

Figure 6 - Scattering of 90 cassava cultivars on a plot defined by discriminant function 1, based on temperature (T1 to T3) of cultivar's place of origin, and discriminant function 2 , based on the first 40 principal coordinate analysis (PcoA) factors. ns, Nonsignificant. *Statistically significant at $5 \%$.

tural practice of mixing several cultivars in the same field (Chernela, 1987; Kerr, 1987), possible recombinations between the different cultivars planted, and crosses with wild species.

Jennings (1963) showed that in the traditional African cassava culture system plants are sexually reproduced by spontaneous germination of seeds in parcels and cloned to generate new genotypes. This phenomenon has been reported in Brazil (Boster, 1984; Kerr, 1987, Martins, 1994). In plants like cassava, the ease of obtaining plants from seed and the ease of vegetative propagation probably accelerated the process of domestication and culture, which consequently encouraged ample variation of morphological features, root quality, etc. This has also been observed in grapes (Alleweldt et al., 1990). Nevertheless, the absence of strong structuring among cultivars from the four geographical groups of this study, with the exception of the Santa Isabel ethnocultivar group, may be explained by transplanting of cultivars as well as genetic recombinations at different sites.

Other factors, such as human migration, can explain the abundance of genetic diversity in other continents. For example, we estimated that half of the 33 million inhabitants of the State of São Paulo immigrated, mostly from the northeastern and central regions of Brazil. These regions are known as the two main centers of genetic diversity of cassava (Nassar, 1978). Part of these migrants were farmers. Cassava was their basic food, and was taken with them when they moved. The diversity revealed by the Santa
Isabel genotypes suggests a certain geographical isolation and limitation of crossing with other cassava cultivars.

The reduced genetic structure observed in relation to the geographical origin of cassava cultivars corroborates the major effects of migration and recombination. As for other vegetatively multiplied species (yam, sweet potato, sugarcane), exchanges of botanical material among producers, which are proportional to the extension of the culture, makes it possible for the same cultivar to be found at several different geographical sites. This is the case with cassava. No one knows the place of origin of this cultivar. A survey of cassava diversity in Africa (Léfèvre, 1993) also revealed little genetic structure in this continent. Léfèvre believed structuring to be associated with the origin of its introduction to the continent, in other words, founder effects. The most recent extension of the cassava culture (beginning about 150 years ago) decreased isolation between parcels and therefore encouraged the flow of genetic material among the different regions.

Nevertheless, cassava structure is associated with its original climate. In this study, cassava groups were differentiated by the precipitation and temperature of their places of origin. Using the variable precipitation, one can see that RAPD markers can separate genotypes based on their adaptability to the availability of water. This tool should help breeders select genotypes appropriate for this type of climate, as suggested by Fukuda et al. (1993) for the semi-arid northeastern region of Brazil.

For the variable temperature, cassava cultures struc- 
tured between $30^{\circ} \mathrm{N}$ and $30^{\circ} \mathrm{S}$. Cordeiro et al. (1995) also observed a climatic adaptation of clones, while forming a core collection as well as assessing the botanical data of 300 cassava clones. According to Carter et al. (1992), African cassava diffused after its introduction to this vast humid tropical zone in the 16th century, because cultivars were well adapted to these specific climatic conditions. Fukuda (1996) stated that the adaptability of the same cassava genotype to a range of different climatic conditions was not frequent, which would partly explain the wide range of genetic diversity of this species.

This study found through RAPD markers that cassava's genetic diversity is great, weakly structured, and tends to structure according to ecological conditions. The manner in which this diversity is distributed in the American continent could be useful for collections and/or conservation of the genetic resources of cassava as well as for breeders. The surprising genetic diversity observed among ethnocultivars from Santa Isabel suggests that the importance of analyzing other peasant communities should be studied.

\section{ACKNOWLEDGMENTS}

The authors gratefully acknowledge CAPES for financial support and fellowships conceded to Carlos Colombo. Publication supported by FAPESP.

\section{RESUMO}

Este trabalho enfoca a diversidade genética de mandiocas americanas através de marcadores moleculares do tipo RAPD. Os 126 genótipos estudados estão distribuídos em quatro escalas geográficas, indo do local ao continental, ou seja, etnocultivares de uma comunidade chamada Santa Isabel, na Amazônia brasileira, cultivares locais coletados no Estado de São Paulo, um grupo representado por acessos oriundos das mais diversas regiões brasileiras e acessos representantes da "core collection" do CIAT. Oitenta e oito bandas RAPD polimórficas foram retidas para as análises. A estrutura genética das mandiocas deste estudo revelou-se fraca. O plano formado pelos dois primeiros eixos da análise de coordenadas principais (PCoA) revelou sobreposição dos genótipos do Estado de São Paulo, do grupo Brasil e dos acessos da "core collection". Por outro lado, os etnocultivares de Santa Isabel mostraram-se estruturados num grupo a parte com relação aos demais genótipos. Além disso, os etnocultivares de Santa Isabel apresentaram importante diversidade genética em relação aos genótipo dos outros três grupos. A fraca estrutura genética das mandiocas cassava pode ser explicada pelas trocas de material botânico entre pequenos produtores, prática normalmente empregada, assim como recombinação entre genótipos, visto que a mistura de diferentes cultivares em uma mesma parcela de cultura é também uma prática usada por pequenos produtores. Entretanto, quando os genótipos foram analisados em função de dados climáticos das localidades de origem dos mesmos, pudemos evidenciar uma estruturação em função da temperatura e da precipitação destes locais. Os marcadores RAPD mostraram-se informativos para o estudo da diversidade genética de mandiocas, oferecendo indicações importantes para a coleta de germoplasma de mandioca, assim como para o seu melhoramento genético. Com relação à importante diversidade genética encontrada na comunidade de Santa Isabel, outros estudos, através de outros marcadores e com genótipos de outras localidades, precisariam ser realizados para se tirarem conclusões mais diretas a respeito desta variabilidade.

\section{REFERENCES}

Alleweldt, G., Spiegel-Roy, P. and Reisch, B. (1990). Grapes (Vitis). In: Genetic Resources of Temperate Fruit and Nut Crops (More, J.N. and Ballington Jr., J.R., eds.). Int. Soc. Hort. Sci., Wageningen., pp. 291-327.

Bonierbale, M.W., Maya, M.M., Claros, J.L. and Iglesias, C. (1995). Application of molecular markers to describing the genetic structure of cassava gene pools. In: The Cassava Biotechnology Network: Proceedings of the Second International Scientific Meeting. Bogor, Indonesia, 22-26 August 1994. Cali, Colombia. Centro International de Agricultura Tropical, 2v. (Working document No. 50).

Boster, J.S. (1984). Classification, cultivation and selection in Aguaruna cultivars of Manihot esculenta (Euphorbiaceae). In: Ethnobotany in the Tropics (Prance, G. and Kallunki, J., eds.). Adv. Econ. Bot. 1:34-47.

Boster, J.S. (1985). Selection for perceptual distinctiveness: evidence from Aguaruna cultivars of Manihot esculenta. Econ. Bot. 39: 310-325.

Carter, S.E., Fresco, L.O., Jones, P.G. and Fairhairn, J.N. (1992). An Atlas of Cassava in Africa: Historical, agroecological and demographic aspects of crop distribution. Centro Internacional de Agricultura Tropical, CIAT, publication No. 206. Cali, Colombia, p. 86.

Chernela, J.M. (1987). Os cultivares de mandioca na área do Uaupés. In: Suma Etnológica Brasileira (Ribeiro B. ed.). Vol. 1. Etnobiologia, Vozes/FINEP, Petrópolis, pp. 159-171.

Cohen, J.I., Alcorn, J.B. and Potter, C.S. (1991). Utilization and conservation of genetic resources: international projects for sustainable agriculture. Econ. Bot. 45: 190-199.

Colombo, C., Second, G., Charrier, A. and Valle, T.L. (1998). Genetic diversity characterization of cassava cultivars (Manihot esculenta Crantz). I. RAPD markers. Genet. Mol. Biol. 21: 105-113.

Cordeiro, C.M.T., Morales, E.A.V., Ferreira, P., Rocha, D.M.S., Costa, I.R.S., Valois, A.C.C. and Silva, S. (1995). Towards a Brazilian core collection for cassava. In: Core Collections of Plant Genetic Resources (Hodgkin, T., Brown, A.D.H., van Hintun, Th.J.L. and Morales, E.A.V., eds.). International Plant Genetic Resources Institute (IPGRI). John Wiley \& Sons, New York, pp.155-169.

Cury, R. (1993). Dinâmica evolutiva e caracterização de germoplasma de mandioca (Manihot esculenta Crantz) na agricultura autóctone do sul do Estado de São Paulo. Master's thesis, ESALQ (Escola Superior de Agricultura Luiz de Queiroz), USP, Piracicaba, SP, Brasil.

De Bruijn, G.H. and Fresco, L.O. (1989). The importance of cassava in world food production. Neth. J. Agric. Sci. 37: 21-34.

Fukuda, W.M.G. (1996). Mandioca: estratégia para um programa de melhoramento genético. EMBRAPA (Empresa Brasileira de Pesquisa Agropecuária). CNPMF (Centro Nacional de Pesquisa em Mandioca e Fruticultura). Documentos 50, p. 35.

Fukuda, W.M.G., Souza, J.S. and Passos, O.S. (1993). Desenvolvimento de germoplasma de mandioca para condições semi-áridas: uma alternativa para o nordeste brasileiro. EMBRAPA (Empresa Brasileira de Pesquisa Agropecuária). CNPMF (Centro Nacional de Pesquisa em Mandioca e Fruticultura). Documentos 50: 5-18.

Hershey, C.H. (1994). Manihot genetic diversity. In: International Network for Cassava Genetic Resources. Report of the First Meeting of the International Network for Cassava Genetic Resources, CIAT, Cali, Colombia, 18-23 August 1992. International Crop Network Series No. 10. International Plant Genetic Resources Institute, Rome, Italy, pp. 111-134.

Hotelling, H. (1935). The most predictable criterion. J. Psychol. 26: 139-142.

Jeffrey, C. (1968). Systematic categories for cultivated plants. Taxon 17: 109-114.

Jennings, D.L. (1963). Variation in pollen and ovule fertility in varieties of cassava and the effect of interspecific crossing on fertility. Euphytica 12: 69-76 
Kerr, W.E. (1987). Agricultura e seleções genéticas de plantas. In: Suma Etnológica Brasileira (Ribeiro, B., ed.). Vol. 1. Etnobiologia, Vozes/ FINEP, Petrópolis, pp. 252-261.

Léfèvre, F. (1993). Isozyme diversity within African Manihot germ plasm. Euphytica 66: 171-178.

Marmey, P., Beeching, J.R., Hamon, S. and Charrier, A. (1994). Evaluation of cassava (Manihot esculenta Crantz) germ plasm collections using RAPD markers. Euphytica 74: 203-209.

Martins, P.S. (1994). Biodiversity and Agriculture: Patterns of domestication of Brazilian native plant species. An. Acad. Bras. Cienc. 66 (Suppl. 1): 219-224.

Nassar, N.M. (1978). Conservation of the genetic resources of cassava (Manihot esculenta). Determination of wild species localities with emphasis on probable origin. Econ. Bot. 32: 311-320.

Nei, M. (1973). Analysis of genetic diversity in subdivided populations. Proc. Nat. Acad. Sci. USA 70: 3321-3323.

Pasteur, N., Pasteur, G., Bonhome, F., Catalan, J. and Briton Davidian, J. (1987). Manuel Technique de Génétique par Électrophorèse des Protéines. Technique et Documentation (Lavoisier), Paris, p. 221.

Pereira, A.V., Vencovsky, R. and Cruz, C.D. (1992). Selection of botanical and agronomical descriptors for the characterization of cassava (Manihot esculenta Crantz) germplasm. Rev. Bras. Genet. 15: 115-124.

Ramirez, H., Hussain, A., Roca, W. and Bushuk, W. (1987). Isozyme electrophoregrams of sixteen enzymes in five tissues of cassava
(Manihot esculenta Crantz) varieties. Euphytica 36: 39-48.

Rogers, D.J. (1972). Some further considerations on the origin of $M$ esculenta. Trop. Root Tuber Crops Newslett. 6: 4-10.

Rohlf, F.J. (1993). NTSYS-pc. Numerical Taxonomy and Multivariate Analysis System. Version 1.80. Exeter Software, Setauket, NY.

Sauer, J. (1952). Agricultural Origins and Dispersals. American Geographical Society, New York.

Schmidt, C.B. (1951). A Mandioca, Contribuição para o Conhecimento de sua Origem. Boletim da Agricultura, Campinas, No. 1:1.

Sokal, R. and Michener, C.D. (1958) A statistical method for evaluating systematic relationships. Univ. Kansas Sci. Bull. 38: 1409-1438.

Ugent, D., Pozorski, S. and Pozorsk, T. (1986). Archaeological manioc (Manihot) from coastal Peru. Econ. Bot. 40: 78-102.

Van de Ven, W.T.G. and McNicol, R.J. (1995). The use of RAPD markers for the identification of Sitka spruce (Picea sitchensis) clones. Heredity 75: $126-132$

Wachira, F.N., Waugh, R., Hackett, C.A. and Powell, W. (1995). Detection of genetic diversity in tea (Camellia sinensis) using RAPD markers. Genome 38: 201-210.

Wilde, J., Waugh, R. and Powell, W. (1992). Genetic fingerprinting of Theobroma clones using randomly amplified polymorphic DNA markers. Theor. Appl. Genet. 83: 835-838.

(Received April 27, 1998) 
